\title{
Cooperative Localization of Drones by using Interval Methods
}

\author{
Ide-Flore Kenmogne, Vincent Drevelle, and Eric Marchand*
}

\begin{abstract}
In this article we address the problem of cooperative pose estimation in a group of unmanned aerial vehicles (UAVs) in a bounded-error context. The UAVs are equipped with cameras to track landmarks, and with a communication and ranging system to cooperate with their neighbors. Measurements are represented by intervals, and constraints are expressed on the robots poses (positions and orientations). Pose domains subpavings are obtained by using set inversion via interval analysis. Each robot of the group first computes a pose domain using only its sensors measurements. Then, through position boxes exchanges, the positions are cooperatively refined by constraint propagation in the group. Results with real robot data are presented, and show that the position accuracy is improved thanks to cooperation.
\end{abstract}

Keywords: cooperative localization, intervals, vision, constraint propagation

\section{Introduction}

Unmanned aerial vehicles (UAVs) have significantly attracted the attention and interest in several applications areas involving multi-robot tasks, such as search and coverage missions [18, 4], exploration [10], cooperative manipulation [20, 11], control $[15,25]$, etc. In each of these cases, one problem to be solved is the cooperative localization (CL) in a group of robots [23]. CL consists in improving the positioning capacity of each robot through information exchanges (e.g. sensors measurements) with other robots in the group.

Several methods exist for solving CL, such as the Extended Kalman Filter (EKF) for a centralized system $[12,22,8]$ or, if the computation is decentralized and the communication is unreliable, other techniques like Covariance Intersection [27] or Interleaved Update [1]. Approaches that assume bounded errors using polytopes and linear programming algorithms have also been proposed in $[26,5]$.

In a bounded-error measurements context, interval set-membership methods [21] allow rigorous errors bounds propagation in non-linear estimation problems.

\footnotetext{
*Univ Rennes, Inria, CNRS, IRISA, E-mail: vincent.drevelle@irisa.fr
} 
Many applications of these methods are found in practical areas like mobile robots localization [7] or positioning integrity for autonomous cars [9], and as part of the cooperative localization of underwater robots with sonar [3] and vehicle networks with GNSS [17]. Interval methods also enable to derive efficient particle filter implementations for highly non-linear localization problems [19]. In [16], the authors have shown that interval methods are an effective tool for vision-based boundederror positioning of a drone.

In this paper, we propose to use interval set-membership methods as a tool to solve the problem of cooperative localization of a fleet of drones. Each drone in the group is equipped with a camera able to track georeferenced landmarks, and a communication and ranging system providing each robot a way to exchange data and measure distances with its neighbors. Communication and ranging are also available with a base station of known position. The aim is to calculate a pose domain for each robot from constraint propagation and set inversion techniques. The proposed method computes, for each drone, a set that contains all its feasilble poses. The solution sets take into account image measurements, distance measurements and the georeferenced landmarks positions; all being uncertain with bounded errors. The computed pose domain of each robot is guaranteed to contain the solution as long as the measurements error bounds are not violated. The paper is structured as follows: Section 2 presents the problem statement, then the proposed estimation method is presented in Section 3. Finally, experimental results are reported in Section 4.

\section{Problem statement}

Let us consider the global reference frame $\mathcal{F}_{w}$, in which evolves a fleet of $n$ drones $R_{k}(k \in\{1 \ldots n\})$. A reference frame $\mathcal{F}_{r_{k}}$ (also known as body frame) is attached to the center of gravity of each robot. The pose of a drone $R_{k}$ is given by $\mathbf{r}_{k}=\left(x_{k}, y_{k}, z_{k}, \phi_{k}, \theta_{k}, \psi_{k}\right)$ in the global reference frame.

In the sequel, we consider that the drones are identical in their architecture. They are equipped with calibrated onboard cameras (the intrinsic parameters are known), and the rigid transformation between the camera frame and the robot body frame is known by calibration and represented by the homogeneous rotation and translation matrix ${ }^{c} \mathbf{T}_{r}$. We also suppose, as in [25], that each robot flies in nearhovering regime, thus allowing the precise measurement of roll and pitch angles. The neighborhood of a robot $R_{k}$, i.e. the set of robots it can communicate with, is denoted by $\mathcal{N}\left(R_{k}\right)$.

\subsection{Scenario description}

The navigation environment consists of a base station $B$ (see Figure 1) able to communicate with the drones, and $m$ georeferenced landmarks (for which the positions are known). In order to accomplish its navigation task, each robot of the group must be located relative to $\mathcal{F}_{w}$. Each drone $R_{k}$ is equipped with the following 


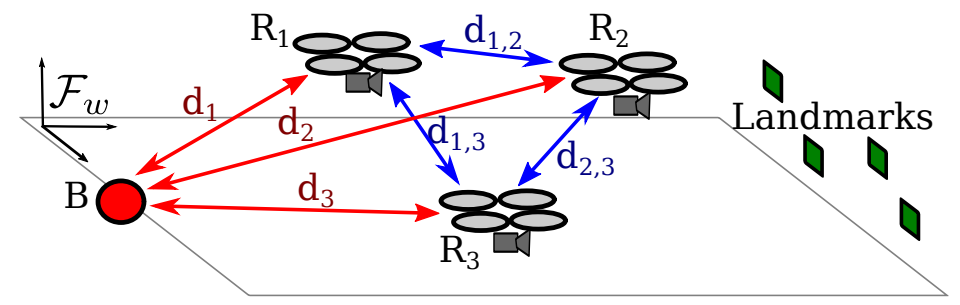

Figure 1: Cooperative localization with camera and range measurements

embedded sensors: camera, altimeter and inertial measurement unit (IMU) with a compass. This enables the following measurements:

- the altitude $z_{k}$;

- the roll $\phi_{k}$ and pitch $\theta_{k}$ angles, with good precision;

- the yaw angle $\psi_{k}$ (with rough precision due to magnetic disturbances);

- and the 2-D points $\overline{\mathbf{x}}_{1}^{k}, \ldots, \overline{\mathbf{x}}_{m}^{k}$ in the image $I_{k}$, corresponding to the observable 3-D landmarks ${ }^{w} \mathbf{X}_{1}^{k}, \ldots,{ }^{w} \mathbf{X}_{m}^{k}$ in the environment.

In addition to these on-board sensor measurements, each robot $R_{k}$ is able to:

- measure its distance $d_{k, j}$ to a neighboring robot $R_{j}\left(R_{j} \in \mathcal{N}\left(R_{k}\right)\right)$,

- measure its distance $d_{k}$ to the base station $B\left(\mathbf{b}=\left(x_{B}, y_{B}, z_{B}\right)^{\top}\right.$ in $\left.\mathcal{F}_{w}\right)$

- communicate and exchange information with each robot in its neighborhood.

This can be done by using for instance an IEEE 802.15.4.a Ultrawide Band (UWB) communication system [24] which allows time-of-flight ranging.

Under the assumption of bounded-error measurements, we want to compute the domains of all the feasible 6-DOF poses for each drone in the global reference frame $\mathcal{F}_{w}$. The components of the pose are those of the position vector $\mathbf{p}_{k}=\left(x_{k}, y_{k}, z_{k}\right)$ and the attitude (roll, pitch and yaw) $\mathbf{q}_{k}=\left(\phi_{k}, \theta_{k}, \psi_{k}\right)$. Given that three of the six degrees of freedoms are measured with low uncertainty $\left(z_{k}, \phi_{k}, \theta_{k}\right)$, our attention will be focused on estimating the horizontal position $\left(x_{k}, y_{k}\right)$ and the yaw angle $\psi_{k}$ of each robot. The knowledge on the altitude, the roll and the pitch measured for each robot will be integrated into the problem in the form of constraints. The set of observation equations allows us to define, for each robot, a set of constraints described in the following section.

\subsection{Set of constraints related to the measurements}

To solve the problem described in the previous scenario, let us express it as a constraint satisfaction problem (CSP). We will then be able to use the SIVIA [13] branch-and-bound algorithm with contractors (see Section 2.3) to characterize the 
domain of feasible poses for each robot. For this, each measurement is related to the state of the robot by a constraint in the CSP describing the problem.

\subsubsection{D image measurements constraints}

The pinhole camera model describes the projection of a 3D point in the image plane. In homogeneous coordinates, a world point ${ }^{w} \mathbf{X}_{i}=(X, Y, Z, 1)^{\top}$ is projected in the image at the pixel coordinates $\overline{\mathbf{x}}_{i}^{k}=(u, v, 1)^{\top}$ given by:

$$
\overline{\mathbf{x}}_{i}^{k}=\mathbf{K} \Pi^{c} \mathbf{T}_{w}\left(\mathbf{r}_{k}\right)^{w} \mathbf{X}_{i}
$$

where $\mathbf{K}$ is the camera intrinsic parameters matrix (principal point coordinates $u_{0}, v_{0}$ and focal length to pixel size ratios $p_{x}$ and $p_{y}$ ) and $\Pi$ is the perspective projection matrix:

$$
\mathbf{K}=\left(\begin{array}{ccc}
p_{x} & 0 & u_{0} \\
0 & p_{y} & v_{0} \\
0 & 0 & 1
\end{array}\right), \boldsymbol{\Pi}=\left(\begin{array}{cccc}
1 & 0 & 0 & 0 \\
0 & 1 & 0 & 0 \\
0 & 0 & 1 & 0
\end{array}\right)
$$

and ${ }^{c} \mathbf{T}_{w}\left(\mathbf{r}_{k}\right)$ is the world to camera rigid transformation matrix, which depends on the robot pose $\mathbf{r}_{k}$ and the known robot to camera transformation ${ }^{c} \mathbf{T}_{r}$.

Let us denote by $\left({ }^{c} X^{k},{ }^{c} Y^{k},{ }^{c} Z^{k}, 1\right)^{\top}={ }^{c} \mathbf{T}_{w}\left(\mathbf{r}_{k}\right){ }^{w} \mathbf{X}_{i}$ the coordinates of a landmark in the coordinate frame attached to the camera of the robot $R_{k}$. We can now define the set of constraints related to measurements in the image:

- The perspective projection constraint of a point in the image $C_{p r o j}^{k}$ :

$$
C_{p r o j, i}^{k}:\left\{\begin{array}{c}
u=p_{x} x_{i}^{k}+u_{0}, \\
v=p_{y} y_{i}^{k}+v_{0}
\end{array}\right\} \text {, with } x_{i}^{k}=\frac{{ }^{c} X^{k}}{{ }^{c} Z^{k}} \text { and } y_{i}^{k}=\frac{{ }^{c} Y^{k}}{{ }^{c} Z^{k}} .
$$

- The front looking camera constraint $C_{\text {front }}^{k}$, ensuring that the landmark is in the front half-space of the camera:

$$
C_{\text {front }, i}^{k}:\left\{{ }^{c} Z_{i}^{k}>0\right\} \text {. }
$$

The pose of each drone $R_{k}$ will have to verify the constraints $C_{p r o j, i}^{k}$ and $C_{f r o n t, i}^{k}$ for all $i$ corresponding to the observed landmarks.

\subsubsection{Base station distance constraint}

Considering a measurement $d_{k}$ of the distance to the base station $B$, the constraint $C_{\text {base }}^{k}$ expresses the fact that the Euclidean distance between the robot $R_{k}$ and $B$ is equal to the measured distance. Recalling that the position part of the robot pose is $\mathbf{p}_{k}=\left(x_{k}, y_{k}, z_{k}\right)^{\top}$, we have :

$$
C_{\text {base }}^{k}:\left\{d_{k}=\left\|\mathbf{p}_{k}-\mathbf{b}\right\|_{2}\right\} .
$$

This constraint is used to reduce the robot's position domain. Indeed, as the position of the base station $\mathbf{b}$ is known, the knowledge of $d_{k}$ constrains the position $\mathbf{r}_{k}$ of the drone to be located on a sphere $S^{2}\left(\mathbf{b}, d_{k}\right)$ as shown in Figure 2. 


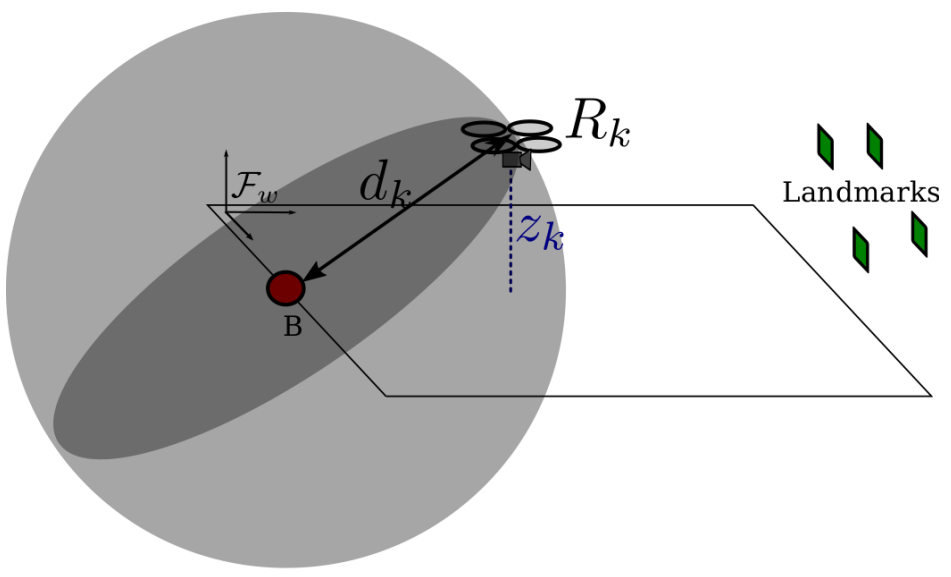

Figure 2: Distance constraint to the base station

\subsubsection{Inter-distance measurements constraints}

Assuming that the ranging system antenna coincides with the center of mass of the drone, distance measurements are independent of the robot attitude. The constraint related to inter-distance measurements between a drone $R_{k}$ and its neighbor $R_{j}$ with $R_{j} \in \mathcal{N}\left(R_{k}\right)$ is defined by:

$$
\begin{gathered}
d_{k, j}=\left\|\mathbf{p}_{k}-\mathbf{p}_{j}\right\|_{2} \quad \text { with }(k, j) \text { in }\{1 \ldots n\}^{2} \text { and } k \neq j, \\
C_{r_{k}, r_{j}}:\left\{d_{k, j}=\left\|\mathbf{p}_{k}-\mathbf{p}_{j}\right\|_{2}\right\}
\end{gathered}
$$

The additional knowledge of $d_{k, j}$ by the robot $R_{k}$ restricts the possible configurations. For a given candidate position of neighbor $R_{j}$, the eligible positions of $R_{k}$ are limited to a circle (intersection of the two spheres $S^{2}\left(\mathbf{b}, d_{k}\right)$ and $S^{2}\left(\mathbf{p}_{k}, d_{k, j}\right)$ ).

Measurements of distances $d_{k}$ and $d_{k, j}$ provide constraints on the robot positions, but there are still 3 degrees of freedom (on 6DoF) corresponding to all rotations of the formation around the base station.

\subsubsection{Altitude measurement constraint}

Let us consider the altitude $z_{k}^{\text {meas }}$ of each robot measured by the altimeter, we have the constraint $z_{k}=z_{k}^{\text {meas }}$ which places $R_{k}$ on a plane.

It allows to constrain the sphere $S^{2}\left(\mathbf{b}, d_{k}\right)$ formed by the distance to the base station $B$ and the robot $R_{k}$ on a horizontal circle resulting from the intersection of $S^{2}\left(\mathbf{b}, d_{k}\right)$ and the plane.

It also allows to constrain the sphere $S^{2}\left(\mathbf{p}_{k}, d_{k, j}\right)$ solution of the measurement of the inter-distances $d_{k, j}$. For a known position of $R_{j}, R_{k}$ has only two possible positions corresponding to the intersection of the horizontal position circle of $R_{k}$ with the sphere $S^{2}\left(\mathbf{p}_{k}, d_{k, j}\right)$. 
Using only distance and altitude measurements leads to an ambiguity on the position of the robot, dependent on the orientation and corresponding to the rotations around the vertical axis of the base station $B$. Adding the constraints from the image measurements will enable to remove the ambiguity.

\subsection{Set inversion via interval analysis with contractors}

The set inversion via interval analysis (SIVIA) algorithm [13] is a branch-andbound method, which enables to compute an outer approximation of the solution of a CSP. The algorithm starts from an arbitrarily big box $\left[\mathbf{x}_{0}\right]$ that must contain the solution set. The solution is then enclosed in an outer subpaving $\overline{\mathbb{S}}$, i.e. a set of non-overlapping boxes, which covers the solution. A parameter $\epsilon$ controls the precision of the obtained solution approximation. SIVIA can be implemented with box reduction operators called contractors [6]. A contractor for a CSP takes an input box, and returns a smaller included box without losing any solution. Assuming a contractor $\mathcal{C}$ is available for the CSP, using for instance interval constraint propagation techniques [2], a very straightforward implementation of SIVIA is given by the following algorithm (see the book [14] for more detailed algorithms):

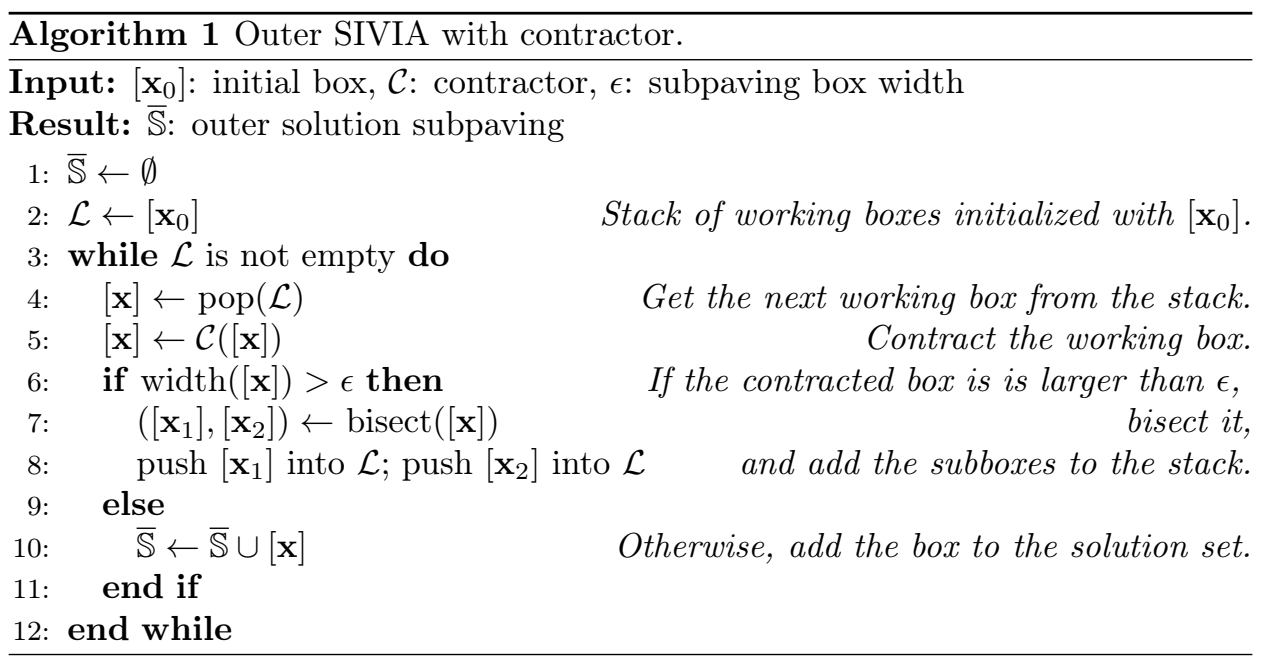

\section{Cooperative localization via position domain ex- change}

From the set of constraints related to the measurements defined in Section 2.2, it is possible to define a CSP which will allow the effective computation of the robot poses domains by propagating the uncertainties on the measurements. We propose a distributed approach that consists in performing a data fusion based on position domains sharing. 
Each robot computes its own pose domain only. In this way, whatever the number $n$ of robots, the number of bisections dimensions in SIVIA remains unchanged (equal to 3 ).

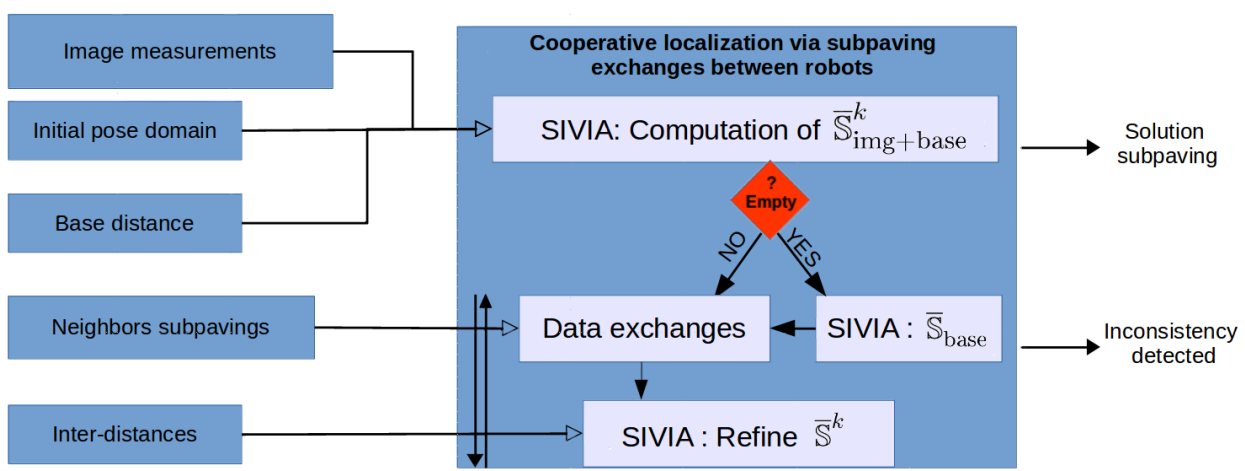

Figure 3: Distributed set-membership pose estimation method.

Position estimation is done in two steps (Figure 3). Firstly, each drone $R_{k}$ ( $k$ in $1 \ldots n$ ) estimates its pose domain by using image measurements and distance to the base station (measurements that depends on robot $R_{k}$ only). Secondly, each drone exchanges its resulting position domain with all its neighbors and, by using inter-distance measurements, refines its position thanks to the constraints of distances between each robot $R_{k}$ and its neighbors $R_{j}, j \in \mathcal{N}(k)$.

To solve the two steps, we define two CSPs for which we characterize a solution set using SIVIA with the HC4 contractor [2].

\subsection{CSP related to vision and distance to the base station}

At each time step, $R_{k}$ first computes an outer approximation $\overline{\mathbb{S}}_{i m g+\text { base }}^{k}$ of the solution set of the CSP $\mathcal{H}_{\text {img }}$ base defined from the constraints of Section 2.2 and given by:

$$
\mathcal{H}_{\text {img+base }}:\left(\begin{array}{rl}
\mathbf{r}_{k} & \in\left[\mathbf{r}_{k}\right], \\
\overline{\mathbf{x}}_{i}^{k} & \in\left[\overline{\mathbf{x}}_{i}^{k}\right], \\
{ }^{w} \mathbf{X}_{i}^{k} & \in\left[{ }^{w} \mathbf{X}_{i}^{k}\right], \\
d_{k} & \in\left[d_{k}\right], \\
\left\{C_{\text {proj }, i}^{k}, C_{\text {front }, i}^{k}\right. & \left., C_{\text {base }}^{k}\right\}, i \in 1 \ldots m
\end{array}\right) .
$$

Adding the distance measurement to the base station makes it possible to refine the position of the robot. We can observe this tightening of the bounds through the result of Figure 4 where a drone observes five landmarks and has a measurement of its distance to the base station. Figure 4a shows the subpaving result for the image measurements only. Figure $4 \mathrm{~b}$ shows the result with measurement in the image and distance to the base station. 


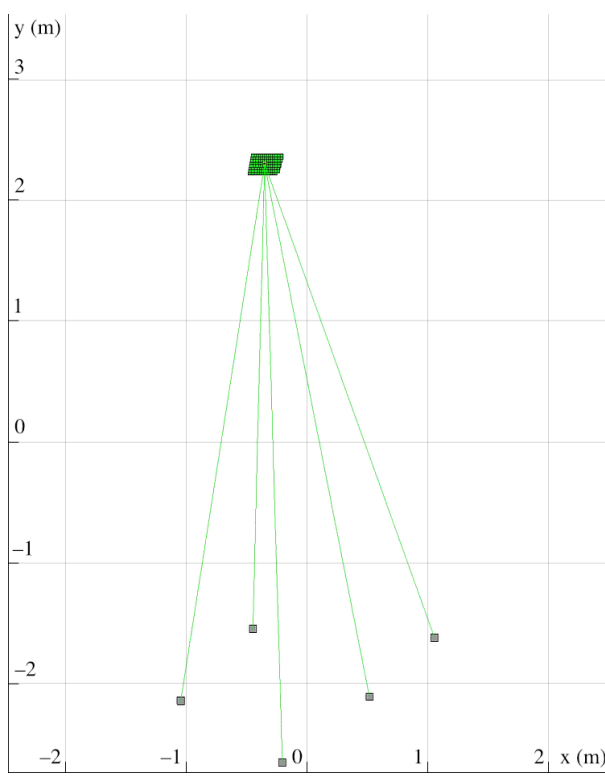

(a) $(x, y)$-plane projection of one robot pose domain with only camera measurements.

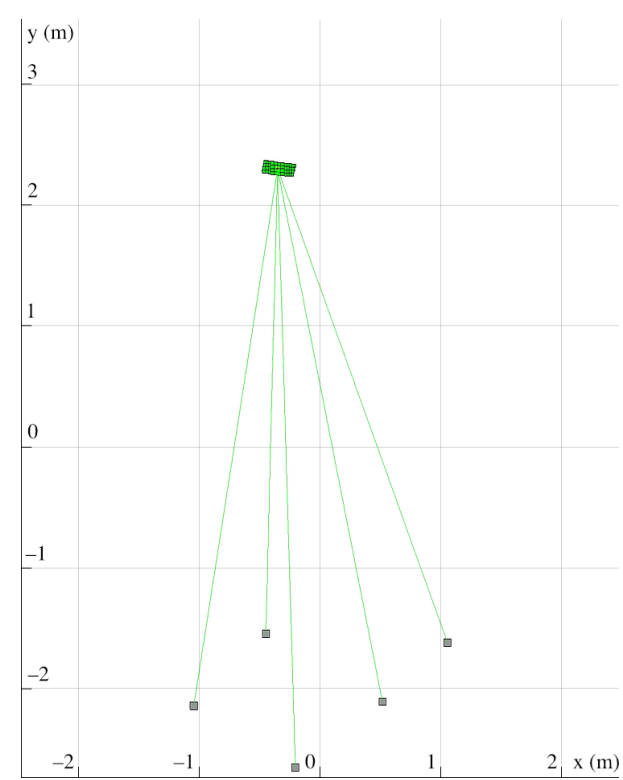

(b) $(x, y)$-plane projection of one robot pose domain with camera and distance measurement to the base station $B$.

Figure 4: Single drone pose domain computation (horizontal projection).

\subsection{CSP related to inter-distance measurements for cooper- ation}

After computing the set $\overline{\mathbb{S}}_{\text {img }}^{k}$ base of poses that are compatible with the distance to the base station and the image, $R_{k}$ computes the bounding box $\left[\mathbf{p}_{k}\right]$ of its position domain:

$$
\left[\mathbf{p}_{k}\right]=\square \operatorname{proj}_{\mathbf{p}}\left(\overline{\mathbb{S}}_{\text {img base }}^{k}\right)
$$

where $\square$ is the bounding box operator and $\operatorname{proj}_{\mathbf{p}}$ the projection of the pose domain on the position space:

$$
\begin{aligned}
\operatorname{proj}_{\mathbf{p}}: & S E(3) \rightarrow \mathbb{R}^{3} \\
& (x, y, z, \phi, \theta, \psi)^{\top} \mapsto(x, y, z)^{\top} .
\end{aligned}
$$

The domain $\left[\mathbf{p}_{k}\right]$ is then shared to all the neighbors $R_{j}, j \in \mathcal{N}(k)$ of the robot $R_{k}$, and the distances $d_{k, j}$ between $R_{k}$ and $R_{j}$ are simultaneously measured.

\subsubsection{Pose domain contraction}

After receiving information from its neighbors (position boxes $\left[\mathbf{p}_{j}\right]$ and distance measurements $\left.\left[d_{k, j}\right]\right)$, each drone $R_{k}$ tries to refine the current bounds $\left(\overline{\mathbb{S}}_{\text {img }}^{k}+\right.$ base $)$ 
of its pose domain. It propagates the information on new inter-distance measurements by locally contracting the CSP $\mathcal{H}_{\text {inter }}$ defined as follows:

$$
\mathcal{H}_{\text {inter }}:\left(\begin{array}{l}
\mathbf{r}_{k} \in \overline{\mathbb{S}}_{\text {img }}^{k} \text { base } \\
\mathbf{p}_{k}=\operatorname{proj}_{\mathbf{p}}\left(\mathbf{r}_{k}\right), \\
\mathbf{p}_{j} \in\left[\mathbf{p}_{j}\right], j \in \mathcal{N}(k) \\
d_{k, j} \in\left[d_{k, j}\right], j \in \mathcal{N}(k) \\
d_{k, j}=\left\|\mathbf{p}_{k}-\mathbf{p}_{j}\right\|_{2} j \in \mathcal{N}(k)
\end{array}\right)
$$

where $\mathbf{p}_{k}=\left(x_{k}, y_{k}, z_{k}\right)^{\top}$ is the position of $R_{k}$. The pose domain $\overline{\mathbb{S}}_{\text {img }}^{k}+$ base is thus reduced by using interval constraint propagation to contract $\mathcal{H}_{\text {inter }}$ and provide the final solution set $\overline{\mathbb{S}}^{k}$.

\subsubsection{Propagation of the position domain improvement}

The constraint network formed by the group of robots can contain cycles covering several robots. The contraction of the local CSPs $\mathcal{H}_{\text {inter }}$ must be propagated again across the network to improve the reduction of the feasible pose domain of each robot.

If, after solving $\mathcal{H}_{\text {inter }}$ the bounding box of the robot's position domain $\left[\mathbf{p}_{k}\right]$ is reduced, $R_{k}$ therefore re-transmits the update of $\left[\mathbf{p}_{k}\right]$ to its neighborhood. This process is iterated until a fixed point is reached, i.e. when there is no significant improvement on the position domains.

The proposed method has been implemented and tested using real data and results are presented in the following section.

\section{Experimental results}

The proposed method has been tested for a fleet of $n=4$ drones, with data acquired on a Parrot AR-Drone2 UAV. The environment contains $m=5$ landmarks, which are boxes on which are placed AprilTag markers [28]. A Vicon motion capture system has been employed to determine the landmarks coordinates, and to provide the ground truth position and orientation of the drone. Figure 5 illustrates the views of the onboard cameras for each drone at time $t=8 \mathrm{~s}$. Error bounds in the image are equal to $\pm 0.5 \mathrm{px}$ and the distance measurement error is assumed to be less than $\pm 5 \mathrm{~cm}$.

\subsection{Influence of cooperation on the size of the pose domain}

The acquired dataset enables to test our method for a different number of drones. Figure 6 shows in green the position subpaving obtained for the drone $R_{1}$ at time $t=6.3 \mathrm{~s}$ in the different cases, from $n=1$ ( $R_{1}$ alone, no cooperation) to $n=4$ (the full fleet cooperates). 

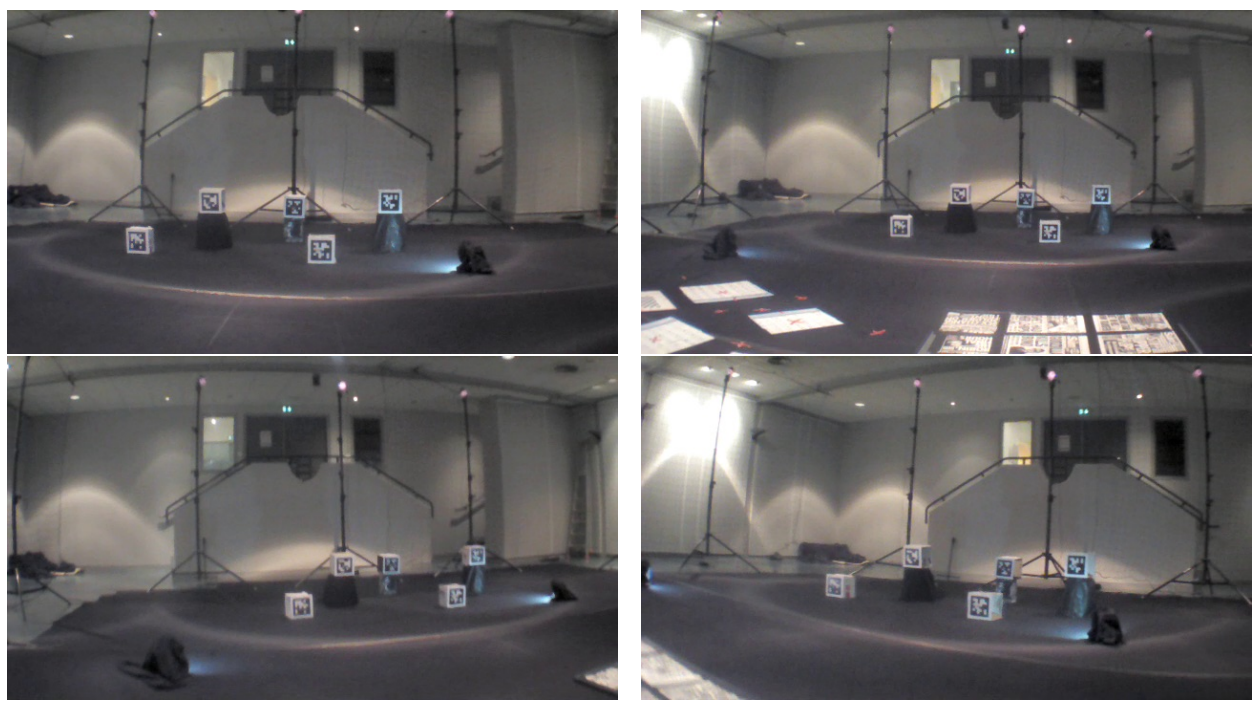

Figure 5: Onboard cameras views at $t=8 \mathrm{~s}$. Landmarks are boxes with AprilTag printed pattern.

At the measurement epoch of Figure 6, the tracking of the landmarks in the image has completely failed for $R_{1}$, which leads to the violation of the image measurements error bounds. Therefore, in step 1 of the computation, the solution $\overline{\mathbb{S}}_{\text {img }}^{1}+$ base is the empty set.

An inconsistency management test has been integrated to the process so that, if $\overline{\mathbb{S}}_{\text {img }+ \text { base }}^{k}=\emptyset$, a domain $\overline{\mathbb{S}}_{\text {base }}^{k}$ related to the base station distance only is computed. Hence the domain of $R_{1}$ in Figure $6 \mathrm{a}$ is $\overline{\mathbb{S}}_{\text {base }}^{1}$, a thick green arc centered on the position of the base station.

Figure $6 \mathrm{~b}$ shows the position subpaving of the drones $R_{1}$ and $R_{2}$, when $n=2$. We notice the significant reduction of the position domain of $R_{1}$ following the cooperation with $R_{2}$, although $R_{2}$ is not very well located (able to observe only two landmarks). More domain reduction occurs in the cases where $n=3$ and $n=4$. This can be observed in Figure 6c and Figure 6d.

These different figures show that cooperation allows a better localization of each robot, even in the case of full landmark visibility loss (case of the robot $R_{1}$ ). This can be confirmed by the statistics of domain widths and horizontal position errors of the 4 robots for groups of different sizes, computed over the whole dataset and presented in Table 1. The average width of the position domain of the drone $R_{1}$ is $1.29 \mathrm{~m}$ when the latter is located without cooperation. Thanks to the cooperation, the width of its domain can be reduced and reach a value of $33 \mathrm{~cm}$ (in the case of a group of 4 drones), which is a $74 \%$ decrease. The average horizontal position error for $R_{1}$, using the center of the subpaving bounding box as a point estimate, improves from $22.6 \mathrm{~cm}$ to less than $5 \mathrm{~cm}$ (first column of Table $1 \mathrm{~b}$ ). 


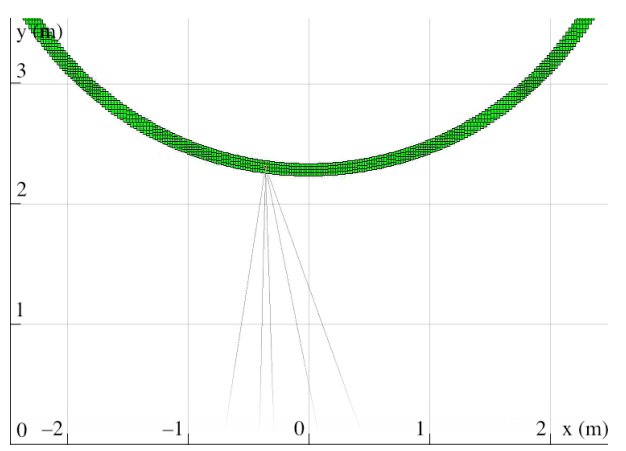

(a) $(x, y)$-plane projection of the pose domain for the drone $R_{1}$ (in green) at time $t=6.3 \mathrm{~s}$

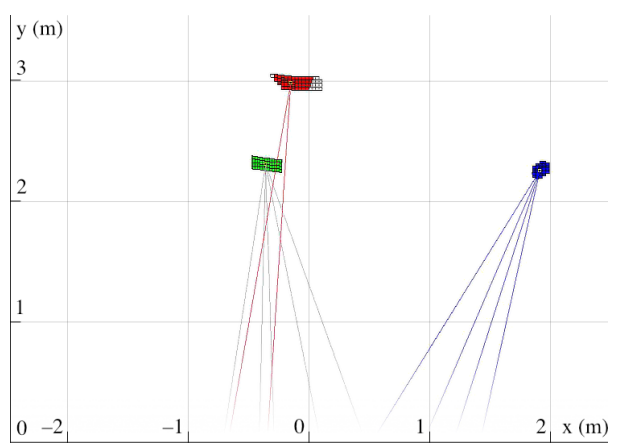

(c) $(x, y)$-plane of the pose domains for drones $R_{1}, R_{2}$ and $R_{3}$ (in blue) at time $t=6.3 \mathrm{~s}$

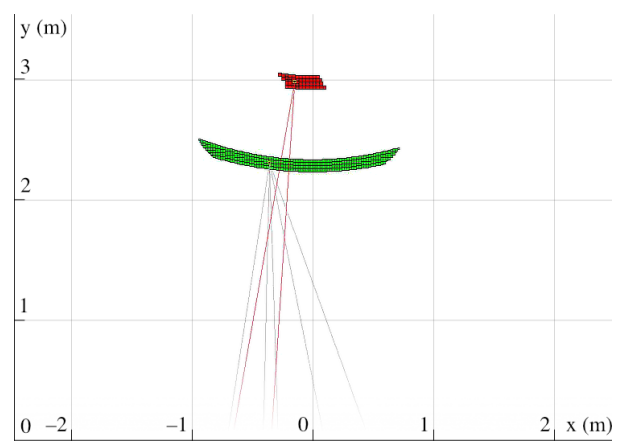

(b) $(x, y)$-plane of the pose domains for drones $R_{1}$ (in green) and $R_{2}$ (in red) at time $t=6.3 \mathrm{~s}$

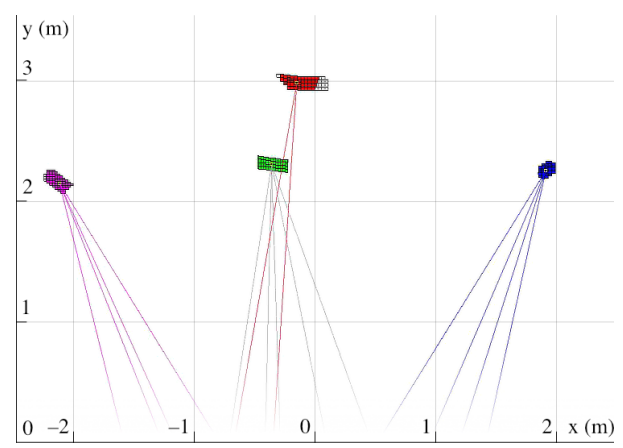

(d) $(x, y)$-plane of the pose domains for drones $R_{1}, R_{2}, R_{3}$ and $R_{4}$ (in pink) at time $t=6.3 \mathrm{~s}$

Figure 6: Horizontal projection of the pose domains computed for an increasing number of robots in the group, at time $t=6.3 \mathrm{~s}$.

With 4 UAVs, the mean horizontal position error of the fleet is about $13 \mathrm{~cm}$.

\subsection{Influence of cooperation in case of reduced visibility}

At certain timestamps of the experiment, the tracking of landmarks in the image fails, thus leading to violations of the error bounds.

Figure 7 presents the subpaving results of our cooperative approach for each of the four drones. The first sub-figure (top-left) presents the case of complete visibility, where each of the drones is able to track the 5 landmarks. The other three sub-figures correspond to cases of reduced visibility where some of the 4 robots were not able to track any point in the image. Black outlines represent the subpavings obtained before cooperation. In case of inconsistency in the image measurements, they are discarded and only the distance to the base station is used to compute the black subpavings. The colored subpavings represent the results 
Table 1: Horizontal position results statistics, for groups of 1 to 4 drones. The reported figures are mean values over the whole experiment.

(a) Mean width of the horizontal position domain bounding box (in meters)

\begin{tabular}{|l|l|l|l|l|}
\hline & $R_{1}$ & $R_{2}$ & $R_{3}$ & $R_{4}$ \\
\hline \hline 1 UAV & 1.29 & & & \\
\hline 2 UAVs & 0.86 & 0.56 & & \\
\hline 3 UAVs & 0.80 & 0.52 & 2.98 & \\
\hline 4 UAVs & 0.33 & 0.37 & 0.78 & 0.61 \\
\hline
\end{tabular}

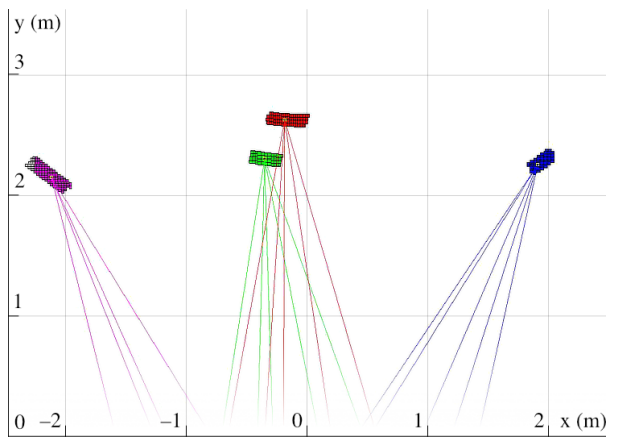

(a) $(x, y)$-projection of the subpavings of the 4 drones in the case of full visibility at $t=0 \mathrm{~s}$

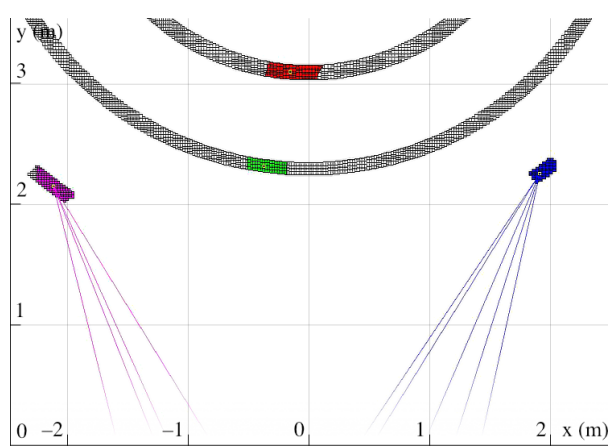

(c) Subpavings of the 4 drones in the case of reduced visibility, $R_{1}$ (in green) and $R_{2}$ (in red) are blind at $t=6.4 \mathrm{~s}$. (b) Mean horizontal position error, considering the center of the bounding box (in meters)

\begin{tabular}{|l|l|l|l|l|}
\hline & $R_{1}$ & $R_{2}$ & $R_{3}$ & $R_{4}$ \\
\hline \hline 1 UAV & 0.23 & & & \\
\hline 2 UAVs & 0.16 & 0.09 & & \\
\hline 3 UAVs & 0.14 & 0.07 & 1.22 & \\
\hline 4 UAVs & 0.04 & 0.05 & 0.25 & 0.19 \\
\hline
\end{tabular}

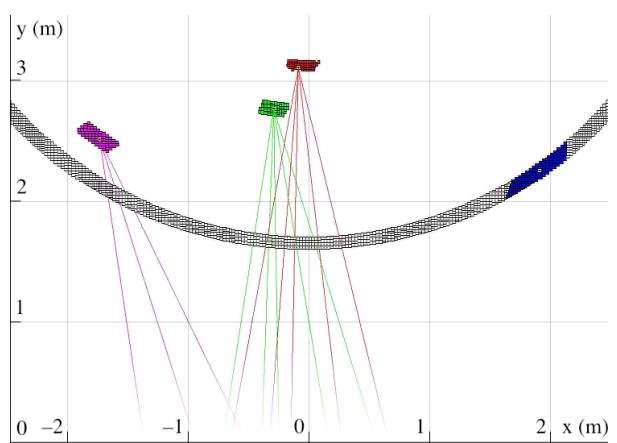

(b) $(x, y)$-projection of the subpavings of the 4 drones in the case of reduced visibility $\left(R_{3}\right.$, in blue, is blind) at $t=14.8 \mathrm{~s}$.

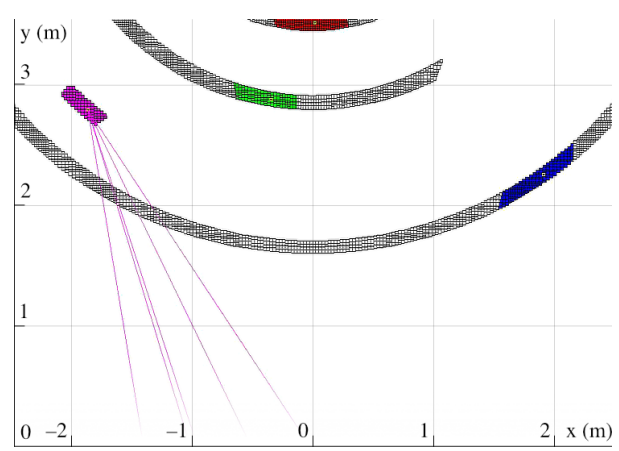

(d) Subpavings of the 4 drones in the case of reduced visibility, $R_{1}$ (in green), $R_{2}$ (in red) and $R_{3}$ (in blue) are blind at $t=12.2 \mathrm{~s}$.

Figure 7: Position domains of the 4 drones. Black outlines: subpavings before robot cooperation. Colored domains: subpavings obtained after cooperative localization. 
obtained for each robot after a fixed point has been reached at the cooperation stage.

These results show that if one of the drones is well located, the cooperation step allows to find an acceptable position domain for all its neighbors.

\section{Conclusion}

In this article, an interval analysis based approach to solve the problem of cooperative localization for a group of unmanned aerial vehicles has been proposed. The fleet of drones use cameras to track visual landmarks, and a communication and ranging system for cooperation. The approach is distributed and based on position domains exchanges between robots. We have shown that using cooperation and domain exchanges makes the approach extensible with respect to the number of robots in the group without a significant computational cost in the estimation process.

Experimental results with real image data have been presented for a group of 4 robots, with an average computation time for each of the drones equal to $350 \mathrm{~ms}$ without having implemented code optimization. Additionally, the results show that cooperation between robots is an asset for group operation when many robots have reduced visibility or are completely blind.

The proposed method can deal with detectable erroneous measurements in the images, i.e. outliers leading to an empty-set solution. It then only relies on distances measurements and data exchange with other robots for position determination. This basic fault detection scheme however does not prevent the propagation of non-detectable image measurements faults to the whole fleet, nor the mitigation of faulty distance measurements. Future work will aim towards the development of adding more fault-tolerance and robustness to interval-based distributed cooperative localization systems.

\section{References}

[1] Bahr, Alexander, Leonard, John J., and Fallon, Maurice F. Cooperative localization for autonomous underwater vehicles. The Int. Journal of Robotics Research, 28(6):714-728, 2009. DOI: 10.1177/0278364908100561.

[2] Benhamou, Frédéric, Goualard, Frédéric, Granvilliers, Laurent, and Puget, Jean-François. Revising hull and box consistency. In Int. Conf. on Logic Programming, pages 230-244. MIT press, 1999. DOI: $10.7551 / \mathrm{mitpress} / 4304.003 .0024$.

[3] Bethencourt, Aymeric and Jaulin, Luc. Cooperative localization of underwater robots with unsynchronized clocks. Paladyn, Journal of Behavioral Robotics, 4(4):233-244, 2013. DOI: $10.2478 / \mathrm{pjbr}-2013-0023$. 
[4] Bevacqua, Giuseppe, Cacace, Jonathan, Finzi, Alberto, and Lippiello, Vincenzo. Mixed-initiative planning and execution for multiple drones in search and rescue missions. In Twenty-Fifth International Conference on Automated Planning and Scheduling, pages 315-323, 2015.

[5] Camillo Jose Taylor, John Spletzer. A bounded uncertainty approach to cooperative localization using relative bearing constraints. In 2007 IEEE/RSJ International Conference on Intelligent Robots and Systems, pages 2500-2506, October 2007. DOI: 10.1109/IROS.2007.4399398.

[6] Chabert, Gilles and Jaulin, Luc. Contractor programming. Artificial Intelligence, 173(11):1079-1100, 2009. DOI: 10.1016/j.artint.2009.03.002.

[7] Colle, E. and Galerne, S. Mobile robot localization by multiangulation using set inversion. Robotics and Autonomous Systems, 61(1):39-48, January 2013. DOI: $10.1016 /$ j.robot .2012 .09 .006 .

[8] Davis, Robert B. Applying cooperative localization to swarm UAVs using an extended Kalman filter. Technical report, Naval Postgraduate School Monterey CA Dept of Computer Science, Sep 2014.

[9] Drevelle, V. and Bonnifait, P. Localization confidence domains via set inversion on short-term trajectory. IEEE Trans. on Robotics, 29(5):1244-1256, Oct 2013. DOI: $10.1109 /$ TRO 2013.2262776 .

[10] Fox, D., Ko, J., Konolige, K., Limketkai, B., Schulz, D., and Stewart, B. Distributed multirobot exploration and mapping. Proceedings of the IEEE, 94(7):1325-1339, July 2006. DOI: 10.1109/JPROC.2006.876927.

[11] Gioioso, Guido, Franchi, Antonio, Salvietti, Gionata, Scheggi, Stefano, and Prattichizzo, Domenico. The flying hand: A formation of UAVs for cooperative aerial tele-manipulation. In 2014 IEEE International Conference on Robotics and Automation (ICRA), pages 4335-4341. IEEE, May 2014. DOI: 10.1109/ICRA. 2014.6907490.

[12] Huang, Guoquan P, Trawny, Nikolas, Mourikis, Anastasios I, and Roumeliotis, Stergios I. Observability-based consistent EKF estimators for multi-robot cooperative localization. Autonomous Robots, 30(1):99-122, Jan 2011. DOI: $10.1007 / \mathrm{s} 10514-010-9207-\mathrm{y}$.

[13] Jaulin, J. and Walter, E. Set inversion via interval analysis for nonlinear bounded-error estimation. Automatica, 29(4):1053-1064, July 1993. DOI: $10.1016 / 0005-1098(93) 90106-4$.

[14] Jaulin, Luc, Kieffer, Michel, Didrit, Olivier, and Walter, Eric. Applied interval analysis: with examples in parameter and state estimation, robust control and robotics. Springer, London, 2001. DOI: 10.1007/978-1-4471-0249-6. 
[15] Jiang, Jingjing and Astolfi, Alessandro. Shared-control for a UAV operating in the 3D space. In 2015 European Control Conference (ECC), pages 1633-1638. IEEE, July 2015. DOI: 10.1109/ECC. 2015.7330771.

[16] Kenmogne, Ide-Flore, Drevelle, Vincent, and Marchand, Eric. Image-based UAV localization using interval methods. In 2017 IEEE/RSJ International Conference on Intelligent Robots and Systems (IROS), pages 5285-5291, Vancouver, Canada, September 2017. DOI: 10.1109/IROS.2017.8206420.

[17] Lassoued, K., Bonnifait, P., and Fantoni, I. Cooperative localization with reliable confidence domains between vehicles sharing GNSS pseudoranges errors with no base station. IEEE Intelligent Transportation Systems Magazine, 9(1):22-34, Spring 2017. DOI: 10.1109/MITS .2016.2630586.

[18] Maza, Ivan and Ollero, Anibal. Multiple UAV cooperative searching operation using polygon area decomposition and efficient coverage algorithms. In Alami, Rachid, Chatila, Raja, and Asama, Hajime, editors, Distributed Autonomous Robotic Systems 6, pages 221-230, Tokyo, 2007. Springer Japan. DOI: $10.1007 / 978-4-431-35873-2 \_22$.

[19] Merlinge, Nicolas, Dahia, Karim, and Piet-Lahanier, Hélène. A box regularized particle filter for terrain navigation with highly non-linear measurements. 20th IFAC Symposium on Automatic Control in Aerospace (ACA 2016), 49(17):361-366, 2016. DOI: 10.1016/j.ifacol.2016.09.062.

[20] Michael, Nathan, Fink, Jonathan, and Kumar, Vijay. Cooperative manipulation and transportation with aerial robots. Autonomous Robots, 30(1):73-86, Jan 2011. DOI: 10.1007/s10514-010-9205-0.

[21] Moore, R.E. Interval analysis. Prentice Hall, 1966.

[22] Qu, Yaohong and Zhang, Youmin. Cooperative localization against gps signal loss in multiple uavs flight. Journal of Systems Engineering and Electronics, 22(1):103-112, Feb 2011. DOI: 10.3969/j.issn.1004-4132.2011.01.013.

[23] Roumeliotis, S. I. and Bekey, G. A. Distributed multirobot localization. IEEE Transactions on Robotics and Automation, 18(5):781-795, Oct 2002. DOI: 10.1109/TRA. 2002.803461.

[24] Sahinoglu, Z. and Gezici, S. Ranging in the IEEE 802.15.4a standard. In 2006 IEEE Annual Wireless and Microwave Technology Conference, pages 1-5, Dec 2006. DOI: 10.1109/WAMICON.2006.351897.

[25] Schiano, Fabrizio and Giordano, Paolo Robuffo. Bearing rigidity maintenance for formations of quadrotor UAVs. In ICRA 2017 - IEEE Int. Conf. on Robotics and Automation, pages 1467-1474, Singapore, Singapore, May 2017. DOI: 10.1109/ICRA. 2017.7989175. 
[26] Spletzer, John and Taylor, Camillo J. A bounded uncertainty approach to multi-robot localization. In Proceedings 2003 IEEE/RSJ International Conference on Intelligent Robots and Systems (IROS 2003), volume 2, pages 12581264, Oct 2003. DOI: 10.1109/IROS.2003.1248818.

[27] Uhlmann, Jeffrey and Julier, Simon. General decentralized data fusion with covariance intersection. In Handbook of Multisensor Data Fusion, pages 319343. CRC Press, Sep 2008. DOI: 10.1201/9781420053098.ch14.

[28] Wang, J. and Olson, E. AprilTag 2: Efficient and robust fiducial detection. In 2016 IEEE/RSJ International Conference on Intelligent Robots and Systems (IROS), pages 4193-4198, Oct 2016. DOI: 10.1109/IROS.2016.7759617. 